\title{
Téoros
}

Revue de recherche en tourisme

\section{Espaces touristiques en pays froids}

\section{Louis-Edmond Hamelin}

Volume 18, numéro 2, été 1999

La nordicité

URI : https://id.erudit.org/iderudit/1072058ar

DOI : https://doi.org/10.7202/1072058ar

Aller au sommaire du numéro

Éditeur(s)

Université du Québec à Montréal

ISSN

0712-8657 (imprimé)

1923-2705 (numérique)

Découvrir la revue

Citer cet article

Hamelin, L.-E. (1999). Espaces touristiques en pays froids. Téoros, 18(2), 4-9. https://doi.org/10.7202/1072058ar d'utilisation que vous pouvez consulter en ligne.

https://apropos.erudit.org/fr/usagers/politique-dutilisation/ 


\section{A}

\section{ESPACES TOURISTIQUES EN PAYS FROIDS}

\section{Louis-Edmond Hamelin}

Dans l'hêmisphère boréal, la zone nivale attire depuis longtemps touristes, sportifs et artistes. Des gravures datant des années 1500 montrent des Lapons en skis courts, avec lesquels les excursions de chasse ne devaient pas être dépourvues de célébrations ; on peut aussi y voir une préfiguration de certaines activités en motoneige. Au XIX $X^{e}$ siècle, en Finno-Scandie, des passagers visitent le Cap Nord de Norvège. Dans les Alpes, des guides suisses, français, britanniques et autres popularisent des promenades prestigieuses sur des glaciers de vallee. Vers 1930, des touristes survolent les icebergs, tristement célèbres depuis le Titanic, de même que les très curieuses tourbières figurées en forêt boréale. Depuis le milieu du XX $X^{*}$ siècle, se développent les loisirs dans les pays froids, dont au Spitżberg, au Yukon et mêmé sur les fleuves sibériens. La Finlande à Rovaniemi et I'Alaska à North Pole reçoivent les amateurs des royaumes du Père Noël. La plupart des activités se font l'été. Depuis peu, est même apparu un tourisme en Antarctique.

Au Canada du XIX ${ }^{e}$ siècle, le Pré Nord du Saguenay et celui de Charlevoix sont régulièrement visités par bateau. Peu après, le rail transcontinental fait naître les célèbres parcs Banff et Jasper dans les Rocheuses enneigées, ce qui procure émerveillement à tous les pèlerins du Commonwealth. En 1962, the Vision of the North d'un premier ministre fait ressusciter à Dawson City le Grand Théâtre de la glorieuse ère du Klondike. Au cours des années 1970, des articles décrivent le tourisme dans les Territoires du NordOuest, À l'échelle du Canada, dix mégarégions nordiques sont identifiées (Hamelin, $1974: 272-277$ ). Le Moyen Nord du Québec s'intéresse aussi à cette industrie naissante (Robitaille et Lejeune, 1967 ; Jamet, 1980 ; Désy, 1980). Après 1950, des visiteurs empruntent le chemin de fer de Schefferville et la route de Chibougamau ; apparaissent les premières publicités sur le Québec-Labrador. Grâce à quelques initiatives, dont celles d'Henri Jamet, s'ouvrent la région hydroélectrique de la baie de James de même que des aires autochtones. À l'est, environ 700000 personnes se rendent à l'impressionnant barrage Daniel-Johnson dans l'arrière Côte-Nord.

Thèses, articles, périodiques et publicités font connattre bien d'autres faits de loisirs. Cependant, les événements, surtout ponctuels et d'inspiration commerciale, ont précédé la conceptualisation et la planification de l'ensemble des lieux nordiques de mểme que l'évaluation des comportements des promoteurs et des visiteurs.

La contribution suivante touche quelques aspects théoriques et informatifs des pays froids. Seront considérées les notions de nordicile et d'hivernité, des propositions vocabulairiques, l'identification de mégarégions à fréquenter ainsi que la présence qui s'intéresse tant à l'espace qu'aux mentalités pourrait conduire à amplifier, à bonifier et à justifier le tourisme du Nord. autochtone. Une telle démarche réflexive

\section{LE CONCEPT ET LES MOTS DE LA NORDICITÉ}

Le terme de nordicité, mis en circulaiton en 1965, exprime l'état, le degré, la conscience et la représentation d'une territorialité froide à l'intérieur de l'hémisphère boréal'. D'une part, il s'agit d'un terme générique qui colle aux traits de l'Alaska, du Canada, du Groenland, de l'Islande, de la Scandinavie, de la Finlande, de la Russie, ainsi qu'aux mers bordières plus ou moins recouvertes de glace. On note l'état circumterrestre de l'encerclement de la froidure, comme l'avait d'ailleurs annoncé le deuxième sens spatial de l'adjectif souche, soit nordique. D'autre part, l'amplitude du terme caractérise aussi sa dimension thématique ; on dépasse les questions de langues et d'ethnies. La nordicité considère donc tout le Nord et le tout du Nord.

La recherche conduit a la notion du Monde circumnordique $^{2}$. La limite sud de ce dernier varie du $50^{\circ}$ degré de latitude vis-àvis de la baie de James, au $60^{\mathrm{e}}$ au golfe d'Alaska, et même au $70^{\circ}$ au nord de la Norvège côtière (carte 1). Les lieux ne sont done pas très éloignés de maints massifs urbains, pourvoyeurs d'assoiffés de loisirs ; ainsi, les pays faiblement nordiques sont en situation d'attente des clientèles. Attrait supplémentaire, la limite sud du vrai Nord constitue l'une des plus importantes ruptures du peuplement continu autour du globe. Cette limite circumterrestre modulée indique notamment des différences considérables dans la durée de la lumière naturelle des pays d'accueil. L'été. si cette disposition cosmique désavantage le Sud-Est du Canada par rapport à la Norvège septentrionale, il en est tout autrement l'hiver, où les jours y sont plus 
longs ; les provinces de l'Atlantique pourraient profiter de cet atout hivernal dans leur publicité touristique.

Entre la limite sud du vrai Nord et le Pôle, s'étendent quatre méga-ensembles dont les capacités récréatives sont originales :

1. Dans le plus lointain, se trouve l'océan Arctique dont l'ouverture aux touristes se traduit par des survols du Pôle, brefs et coûteux.

2. Le Nord russe, mal équipé d'ailleurs, fut interdit de séjour libre jusqu'à récemment.

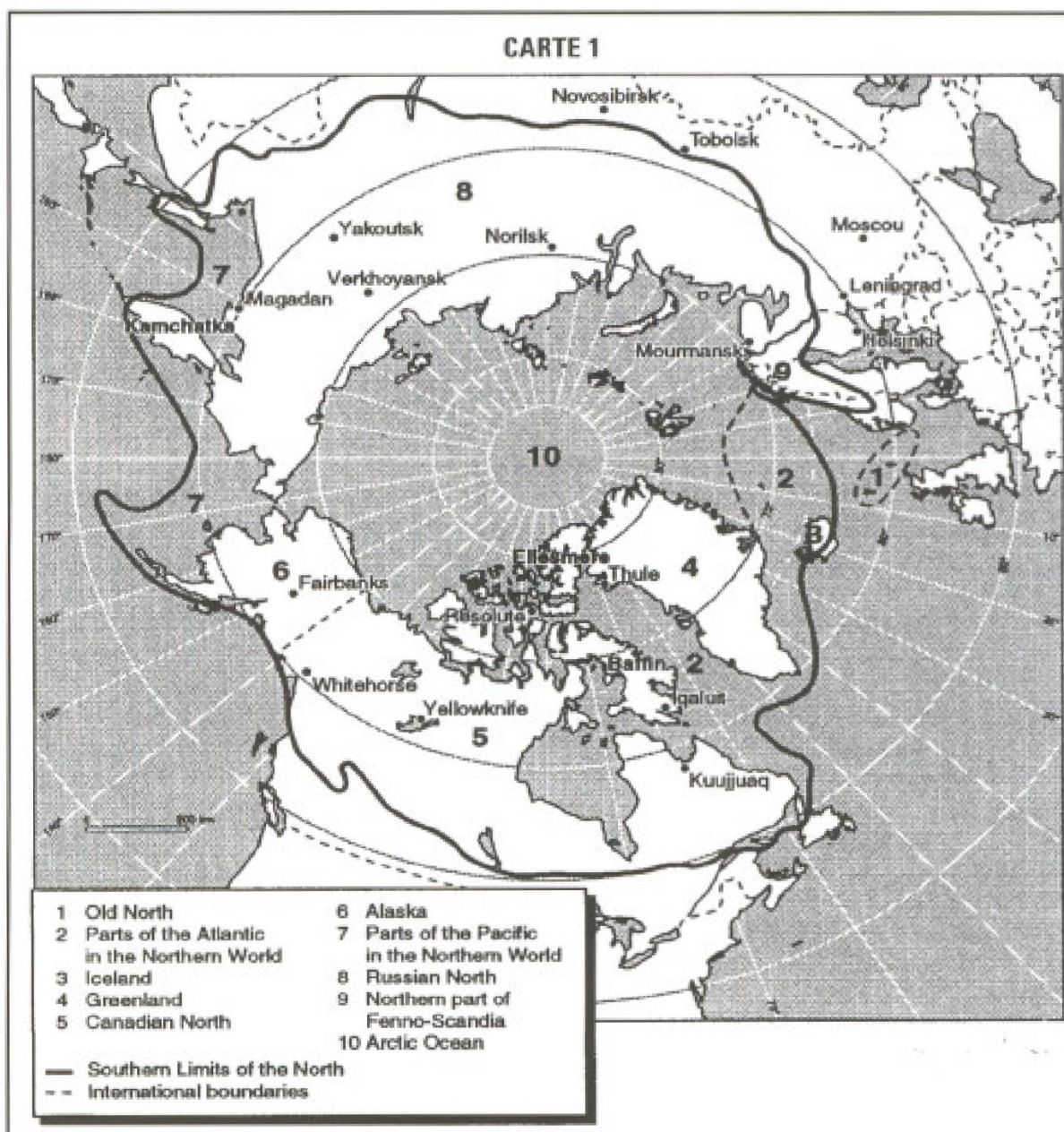

Limite entre le vrai Nord et le Nord périphérique. La limite sud entre le vrai Nord et le Nord périphérique sépare l'immense Monde circumnordique de sa frange prénordique. Cette zone, représentée ici, comprend neuf entités spatiales contemporaines qui ne sont pas également ouvertes au tourisme.

Une vue d'ensemble de l'hếmisphère borếal ferait reconnaitre que l'achalandage touristique est beaucoup plus élevé dans les régions de faible nordicité, durant la saison d'été ainsi qu'en bordure des mers.

Dans le futur certains facteurs, comme l'intérêt à l'endroit du peu connu, le moindre éloignement de la partic Moyen Nord, la stabilité politique et l'absence de terrorisme, pourront jouer en faveur du développement des loisirs à l'intérieur du Monde circumnordique.

Nota : L'écriture des noms de lieux varie.

Saurces: Sommet mondial de la nordicité, Summit on Nordicity, Québec, 1999
À l'intérieur du Monde nordique résident seulement une dizaine de millions d'habitants en permanence, la plupart en Europe, scandinave ou russe. En Amérique, l'immigration est loin d'avoir rattrapé celle qu'ont connue le Norden et l'ex-URSS. Cette présence humaine différentielle correspond au niveau plus élevé de la nordicité moyenne du Canada par rapport à celui des autres contrées nordiques. Par contre, une telle sévérité devient un avantage pour le visiteur du monde qui cherche un dépaysement évalué en fonction d'une faible présence de ses semblables.

On ne visite pas et on ne visitera jamais d'un seul coup la totalité du vrai Nord ; l'exploit n'est même pas réalisable au cours de toute une vie. Mieux vaut done présenter la zone en respectant ses parties. Cependant, cette intention pose un problème de régionalisation. Pour raisons statistiques, la plupart des auteurs considèrent généralement les États indépendants, cantonnés à l'intérieur de leurs frontières nationales. Au contraire, procéder par zone offre le mérite de mieux faire voir la nordicité absolue, les variations latitudinales, ainsi que la distance de la demande sudiste pour chaque niveau de l'offre nordique. Ainsi, trois zones circumpolaires sont identifiées : (du sud au nord) le Moyen Nord [Middle North], le Grand Nord [Far North] et l'Extrême Nord [Extreme North].

La démonstration zonale sera faite à partir de l'exemple canadien [carte 2]. Il s'agit d'abord du Moyen Nord qui constitue la tranche la moins éloignée du Canada de base. Elle correspond à la zone subarctique avec sa forêt boréale et ses tourbières figurées, objet des célèbres fresques de René Derouin. Les mégacentrales d'Hydro-Québec sont des phénomènes remarquables à voir, même à l'échelle mondiale.

Le Grand Nord possède une nordicité une à deux fois plus élevée que celle du Moyen Nord. Il correspond à l'Arctique, sans arbres, avec des nuits fraîches. Par ses chenaux polaires, ce Nord éloigné offre des faciès très différents de ceux auxquels la civilisation occidentale est habituée. Les Inuit sont au Grand Nord ce que les Indiens sont au Moyen Nord.

Enfin, l'Extrême Nord, pays de glace sur mer, sur terre et sous terre, comprend une partie de l'archipel polaire qui donne sur 


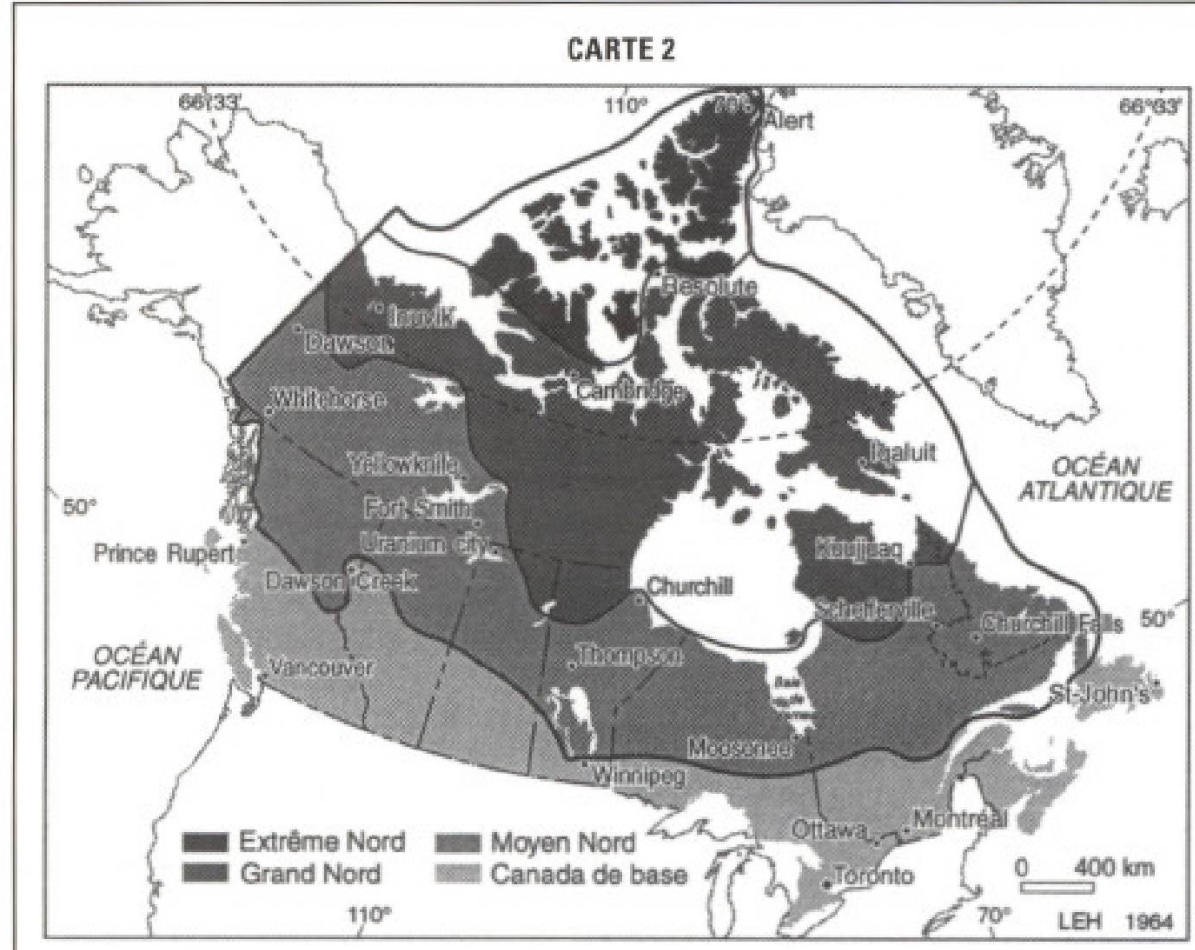

Zones nordiques du Canada. Aux limites du Canada de base, se situe l'aire touristique septentrionale la plus achalandée du pays ; le fait reflète des questions d'intérết, d'étendue et de proximité des grands foyers de peuplement. Cette frange proximale est dénommée Pré Nord et s'étend de l'Atlantique au Pacifique. S'y trouvent de nombreux endroits attractifs dont le Parc Terra Nova, la Gaspésie, le Saguenay, les Laurentides, le Near North ontarien si bien représenté par les tableaux du Groupe des Sept, Minaki, le lac Winnipeg, la rivière Saskatchewan du Nord, de même que Rivière-de-la-Paix en Alberta et en Colombie-Britannique.

Le Moyen Nond, lui, renferme d"attrayantes régions qui fonctionnent surtout l'été. Il en est ainsi de l'ile d'Anticosti, du Parc de la Minganie, des centrales de la Côte-Nord et de la Radissonie (baie de James), des rails de Moosonee et de Churchill, du parc Nahanni et, surtout, de la route vers l'Alaski.

Le long de la côte Atlantique du Grand Nord, on pourrait développer des sites polyvalents d'accueil, par exemple Pangnirtung près du pare national d'Auyuttuq dans le nouveau Nunavut : cette région alpine offre de grandioses attraits : culture inuit, lumiêre ou obscurité au-delà du Cercle polaire, glaciers, fjords, océan, icebergs et versants montagneux vigoureux.

Nota : L'écriture des noms de lieux varie.

Source : Sommet mondial de las nordicité, Summit on Nordicity. Québec, 1999

l'océan Arctique. Les termes d'expédition et d'exploration devraient accompagner celui de simple excursion.

\section{NOTION ET VOCABULAIRE DE L'HIVERNITÉ}

La seconde nordicité circumterrestre, annoncée plus haut, concerne la zone d'hiver à durée limitée (trois à cinq mois), comme à Stockholm, à Moscou ou à Winnipeg. Ici, la nordicité est saisonnière et occupe une position médiane entre la zone précédente de vrai Nord, où l'hiver occupe plus de la moitié de l'année, et la zone des basses latitudes où la saison froide dure moins de soixante jours pour en at- teindre zếro au golfe du Mexique. L'intérêt à l'endroit de cette nordicité temporaire profite d'êvénements ou d'institutions comme l'Association des Villes d'Hiver fondée à Sapporo, le Forum arctique d'Europe de même que le Sommet mondial de la nordicité tenu à Québec. L'hiver, comme le Nord, a fait l'objet de nombreuses études et opinions (Veyret, 1951 ; Deffontaines, 1957 ; Wilson, 1967-69 ; Rudolf, 1981 ; de la Soudière, 1987 ; Collectif, 1998 ; Cazelais, 1998 ; Pressman, 1999 ; Arcand, 1999 ; Association des viles d'hiver, 1999 ; Arseneau, 1999 ; Toupin, 1999).

L'hivemité conceme le fait et le vécu d'une période froide d'une certaine durée $e^{3}$. La notion est liée à l'hiver qui est un phếno- mène socio-météorologique intégré et non seulement une série de manifestations naturelles. $L$ 'hivernant fait indissociablement partie de son propre milieu froid. Autrement dit, l'hivernité prend en compte, d'une part, les traits de l'atmosphère et, d'autre part, les comportements positifs et négatifs des individus.

Le vocabulaire de l'hiver exprime cette proximité de la nature et de la culture, comme le suggère la série de trois déterminatifs dont deux sont des néologismes.

Hivernal pourrait être réservé aux aspects naturels de la saison comme le gel, la neige, la glace, le vent, la poudrerie, le soleil. A prime abord, le qualificatif s'appliquerait à la station étatsunienne Pôle Sud en Antarctique où la moyenne des températures du mois le plus dur se fixe à moins $57^{\circ} \mathrm{C}$, ce qui laisse supposer des jours à moins 70 , même sans vent. Pendant longtemps, beaucoup de chercheurs et de médias en sont restés aux traits physiques dans leur description de l'hiver. Il faut donc identifier un autre mot qu'hivernal, un signe qui représenterait plus que les aspects cosmiques.

Le contenu recherché résiderait-il dans hiverniste? Au contraire du mot précédent, il vise à n'exprimer que les caractères humains, si fortement rendus par les hivernants. Le mot hiverniste, intéressé à l'homme et à son imaginaire, renvoie à une vaste gamme d'attitudes favorables, neutres et, souvent, défavorables; en effet, l'optique hiverniste fait ballotter la saison froide entre un Dies Irae presque révolté et un Magnificat angélique. Qu'un poète fasse savoir qu'hiver rime avec Lucifer, somnifere, impair et colère pendant qu' un autre met en parallèle hivernal et infernal, de tels écrivains énoncent une forte subjectivité. Un autre exemple est celui d'un énoncé emprunté à l'Europe occidentale quand des Québécois parlent de la « grisaille de l'hiver $\%$, alors que la Laurentie, par la neige, éclate de lumière. Les maladresses d'hivernitude vendent plutôt mal l'hiver laurentien!

Dans la présente analyse, voulue utile à une conception attractive de l'hiver, ne fautil pas se rendre encore plus loin ? En effet, le cycle saisonnier complet ne peut s'en tenir aux inconvénients naturels et aux frissons des artistes. Alors, de l'hivernature annoncé par le terme hivernal, de l'hiver-culture véhiculé par celui d'hiver- 
niste, on arrive naturellement à hivernien qui exprime une combinaison, il va de soi, nature. Hivernien renvoie donc au faciès total - physique, humain et régional - de l'hiver. La comparaison entre ces trois notions fait qu'hivernal répond à un état presque sans l'homme, hiverniste, à un contenu construit sans trop de base réelle et hivernien à un phénomène composite socio-météorologique. En d'autres termes, on pourrait réduire hivernal aux stimuli externes, associer hiverniste aux emotions, mais voir dans hivernien l'occasion d'un dialogue nécessaire entre les mondes dits animé et inanimé.

La station antarctique, identifiée précédemment, montre bien les différences entre ces trois etats. Là-bas, les stress les plus gênants ne viennent pas de la froidure ou de la nuit polaire, ce qui consacrerait le poids du concept d'hivernal ; les tensions majeures sont plutôt associées aux facteurs humains, tels l'éloignement, l'exiguïté de l'habitat et la faible variété des loisirs. Dans ces conditions, les travailleurs et les visiteurs mentalement les mieux préparés composent les groupes les moins affectés par la puissante hivernité australe ; c'est qu"ils pratiquaient dejà un comportement hivernien équilibré. Le motif a pas-frileux $x$, de référence proprement hivernale, ne pese guere.

De telles considérations ont leur place dans l'industrie touristique, chez les organisateurs et chez les clients, que les uns et les autres soient dans le vrai Nord ou au Pré Nord, en été ou en hiver. Évidemment, seul l'horizon hivernien, harmonieux comme il l'est par définition, peut bien servir les objectifs, tant de l'offre que de la demande. Ces remarques relevent de la nordicité mentale.

Le tourisme et les sports d' hiver trouvent, dans la glace même, un autre horizon d'activités. Voici deux notions véhiculées par des mots propres.

La première concerne le glaciel ${ }^{4}$ qui se définit par: a tout ce qui concerne les glaces flottantes elles-mêmes et leurs multiples effets dans les domaines tant naturels qu'humains $\nsim$. En particulier, le glaciel offre d'abord une surface de circulation polyvalente, depuis les ponts de glace du debut de la colonie jusqu'aux déplacements en voiture, en traîneau, en skis, en motoneige ou en planche sur des carapaces stables et sans bourguignons (cônes naturels de glace) ; les sportifs qui cherchent davantage de risques tentent des cheminements louvoyants à travers des glaces en mouvement.

En outre, le glaciel est le lieu d'un théatre en plein air où se jouent des spectacles sans cesse renouvelés de son, de lumière et d'action. Côté action, des déplacements massifs de glaçons indisciplinés se produisent tout l'hiver dans les secteurs à marée du Saint-Laurent et durant les débâcles des lacs et des rivières à la fin de la saison. Côté lumière, les glaces mobiles reflètent l'ênergie reçue du soleil et laissent voir une varieté infinie de demi-tons qui, une fois envahis par de légers brouillards, prennent un faciès impressionniste. Côté son, ces mêmes glaçons se fissurent (se gélifractent), s'entrechoquent et se chevauchent en émettant une multitude de bruits dont des quasi-détonations ; les grondements continus se répètent chaque fois que les glaçons changent de voisins, un peu comme dans les conversations sans cesse recommencées des commères. Malheureusement, les visiteurs ne profitent guère de ces manifestations gratuites de la nature : aussi, la suggestion d'élever en proximité des rives des belvéderes confortables d'observations guidées nâtt-elle. Voilà la perspective d'un nouveau tourisme de connaissances et d'émerveillement.

Le second domaine glacique reçoit une double réponse du public. Il s'agit de la glissité qui comporte à la fois plaisir et danger. Elle exprime l'aptitude d'une surface lisse à favoriser le déplacement, désiré ou non, de tout objet situé dessus. D'une part, se trouvent les nombreux sports de glisse que l'on pratique ou que l'on se contente d'observer, comme lors des compétitions. La glisse, elle, constitue une action récréative menée par un individu ou un groupe utilisant toutes les manières de se mouvoir, en piste ou hors piste, sur une surface de neige ou de glace naturelle, artificielle ou composite. D'autre part, la glissité peut notamment se traduire par de la glace noire. Le qualificatif accentue la force de l'enoncé ; il s'agirait d'une très glace dure et polie qui, par grand froid, résiste même aux puissants abrasifs. $\mathrm{Au}$ sens propre, le mot noir rendrait la couleur foncée du support d'une glace fine et transparente, un exemple en est donné par l'asphalte vue à travers une lamelle glacique. Le degré de la glissité varie selon l'état de la surface, la fréquence des passages, l'adresse du marcheur et l'habilité du chauffeur. Bref, si la glissité peut être trailtre, pas seulement pour le touriste sans expérience des pays froids, elle est appréciéc des enfants et des amateurs de bobsleighs ou de courses sur glace.

Les glaces, en particulier par le glaciel et la glissité, se présentent comme des phénomènes importants du tourisme d'hiver.

La saison froide occupe des étendues propres désignées ici d'hivernies (tableau 1). Elles se rattachent à deux grands groupes : I'hivernie matérielle, définie surtout par les stimuli naturels et l'hivernie dérivée, hiverniste, issue des constructions cérébrales. Comme on l'a vu, cette demière correspond au domaine fictif et vient des interprétations de l'hiver.

Du nord au sud, les hivernies spatiales répondent aux zones climatiques polaire, arctique, subarctique, tempérée froide, tempérée chaude et subtropicale. Comme pour le Monde nordique proprement dit, des appellations spécifiques identifient chacune des régions correspondantes d'hiver. En montagne tropicale, les étages froids sont désignés nevada, helada et fria.

L'hivernie intermédiaire, celle du climat tempéré froid, s'amène comme l'aire charnière de tout le monde hivernien circumterrestre. Dans cette hivernité modếrée, mais bien caractếrisếe - vivent des centaines de millions d' habitants dont un certain nombre se transforment en vrais touristes d'êté ou d'hiver. Parmi les multiples sites nordiques visités, se trouvent les côtes de la Baltique, de Norvège, de Kola, d'Islande, d'Alaska et du SaintLaurent. En hiver, les moyennes montagnes de la Scandie et du Japon septentrional de même que les hautes chaînes Alpes, Carpathes, Pyrénées et Cordillères nord-américaines - attirent sportifs et spectateurs de la glisse. Plus spécifiquement, au Canada, les touristes dans les Appalaches, les Laurentides, les Adirondacks, les rebords du Bouclier en Ontario et au Manitoba (Riding), ainsi que ceux des hautes montagnes en Alberta se déploient à un niveau intermédiaire d'hivernité.

Par contre, en hiver, les vacanciers qui s'absentent momentanément des pays tempérés froids choisissent les zones méditerranéenne, subtropicale ou tropicale. Étant donné l'a-nordicité de ces destinations, on devrait qualifier ces demières de 


\begin{tabular}{|l|l|l|c|}
\hline \multicolumn{4}{|c|}{ Zones hiverniennes, hémisphère boréal } \\
\hline climat & $\begin{array}{l}\text { TABLellations des } \\
\text { espaces soumis } \\
\text { à l'hiver }\end{array}$ & $\begin{array}{l}\text { lieux en } \\
\text { Amérique } \\
\text { du Nord }\end{array}$ & $\begin{array}{c}\text { indice hivernité/ } \\
\text { population }\end{array}$ \\
\hline $\begin{array}{l}\text { polaire } \\
\text { arctique } \\
\text { subarct. }\end{array}$ & $\begin{array}{l}\text { hyperhivernie } \\
\text { mégahivernie } \\
\text { hémihivernie }\end{array}$ & $\begin{array}{l}\text { Prudhoe B. } \\
\text { lqaluit } \\
\text { Fairbanks }\end{array}$ & $\begin{array}{l}.0005 \\
1\end{array}$ \\
\hline $\begin{array}{l}\text { modéré ou } \\
\text { p.t.fr. }\end{array}$ & $\begin{array}{l}\text { hivernie } \\
\text { intermédiaire }\end{array}$ & Calgary & 79.8 \\
\hline $\begin{array}{l}\text { p.t.ch. } \\
\text { f.subtr. }\end{array}$ & $\begin{array}{l}\text { infrahivernie } \\
\text { minihivernie }\end{array}$ & $\begin{array}{l}\text { Wash. D.C. } \\
\text { Floride }\end{array}$ & 15 \\
\hline
\end{tabular}

Ce tableau de l'hémisphère boréal apporte, en plus d'une terminologie, des renseignements sur la zonation et le degré de sévérité. On peut voir les choses de plusieurs façons :

Plus d'un milliard de personnes de l'hếmisphère sont touchées au moins par une courte saison froide, ce qui représente de 15 a $20 \%$ de tous les terriens.

Si la majorité des hivernants précédents vivent dans l'hivernie douce des pays tempérés chauds et dans les franges subtropicales, $40 \%$ du milliard d'individus passent néanmoins l'hiver dans la zone de l'hivernie intermédiaire (où le froid se manifeste durant trois à cinq mois).

On peut aussi jumeler le nombre d'habitants avec leur degré d'hiver, en d'autres termes, évaluer l'écoumène de froidure. La démarche fournit un outil d'êvaluation du total des résidents pour tel ou tel niveau d'hivernité ; ici, le trait discriminant est simplement exprimé par le nombre de mois froids dans l'année (indice hivernité). Vu ainsi, à l'échelle de l'intensité de l'hiver, c'est $80 \%$ des valeurs issues de cette combinaison qui se logent dans la zone intermédiaire. De nouveau, celle-ci apparaît done située entre le monde circumnordique peu peuplé et les aires populeuses à faible, voire nulle, hivernité. Plus spécifiquement, les pays tempérés froids (t.fr., au tableau) ont un hiver moins rigoureux que l'hiver sévère des hautes latitudes (polaire, aretique, subarctique), mais plus sérieux que l'hiver léger des basses latitudes (pays tempérés chauds ou t.ch., franges subtropicales ou $\mathrm{f}$. subtr.).

On peut considérer capitales les données de $40 \%$ et $80 \%$ car elles démontrent l'importance de la zone médiane en tant qu'aire de départ et aire d'arrivée touristique.

* tourisme en hiver * plutôt que de * tourisme d'hiver $\%$.

L'hiver se présente comme une saison, un espace, ainsi qu' une émotion. Les exigences de la nordicité mentale et d'une autochtonie raisonnable influencent l'organisation du tourisme des pays froids. Les * Premierres Nations * sont plus que des objets $^{5}$. On ne visite pas une Réserve comme on admire une chute d'eau. Les actes les plus difficiles sont ceux qui se situent à l'interface immédiate des Autochtones et des non-Autochtones. Les relations se font suivant des codes qui ne sont pas exclusivement ceux de visiteurs souvent maladroits. L'autochtonie offre au touriste préparé et attentif plus qu'un dépaysement courant; elle lui laisse voir d'autres façons de vivre, c'est-à-dire autant de visions originales de l'âme.

Dans la majorité des pays froids, l'industrie touristique nordique demeure sous-déve-

\section{NOTES}

1 L' histoire du concept de mondicité touche plusieurs décennies. A la fin des années 1950 . se fait sentir le besoin d'un mot générique se rapportant à I'ensemble du Nord, à la fois comme région et comme sujet pluridisciplinaire. Dans les grands dictionnaires françăis (GDE Larousse, 1963 ; Robert, 1966) et anglais (Webster"s, 1925 ; Oxford, 1933), les mots nordicité et nordiciry ne paraissent pas. C'est que la notion globale ne fut créce qu'en 1963 Yellowknife, puis présentée au congrès de L'Association canadienne des géographes (1965a) et publiée dans la Revue de Geographie Alpine (Association canadienne des géographes, 1965b). Le concept se développe surtout au Centre d'études nordiques de I'Université Laval ; à Montréal, il fait l'objet d'un hardi titre d' ouvrage chez HMH en 1975 et, en anglais, chez Harvest House quatre ans après. Au cours de la décennie 1980, il entre dans les grands dictionnaires de Paris et, en 1998, dans le Canadian Oxford Dictionary. En 1999, à Québec, il sert de signe de ralliement au Sommet mondial de la nordicite. Progressivement, nordicite devient tronc de provignement pour plus de 150 entités dont nordicité mentale, nordicité saisonnière et nordicite zonale, dérives d'ailleurs expliqués dans le présent texte. Par ailleurs, plus de 1100 termes nord et hiver sont consignés dans le manuscrit de LouisEdmond Hamelin, Le Quebec par des mots (Hamelin, n.p.).

2 Afin de localiser l'entièreté et les zones du Nord géographique, outil et table de calcul sont mis au point en 1963-64. Hamelin, Louis-Edmond (1968), a Un indice polaire : Annales de géographie, 422, p. 414-430. Première version anglaise dans W.C. Wonders (ed.) (1971), Canada's Changing North, Toronto, MCS, p. 7.21 (la mesure du Nord géographique s'exprime en vapos, abréviation pour *valeurs polairess; ; dans l'hémisphère boréal, un vapo est le millième de la nordicité au Pôle Nord).

3 Une définition élaborée de l'hiver, suivant une conception nature pourrait être la suivante : période froide, nivale et glacique des interfaces air-terre-mer, variable suivant les types de temps, les lieux, les jours et les années, ainsi qu'influencée par les niveaux techniques, les sports, les services publics, la pression sociale et le comportement des individus.

4 Le mot glaciel, mis en circulation en 1959 pour glaces flottantes, apparait aे la Gazette officielle du Québec (1980), au Grand Robert et au Petit Larousse ; il possède deux entrées dans la toponymie du Québec. Par ailleurs, la famille du tronc glaciel comprend au moins une centaine de termes provignếs,

5 Autochtones: groupe d'individus, nation ou peuple, de souche antérieure aux colonisa- 


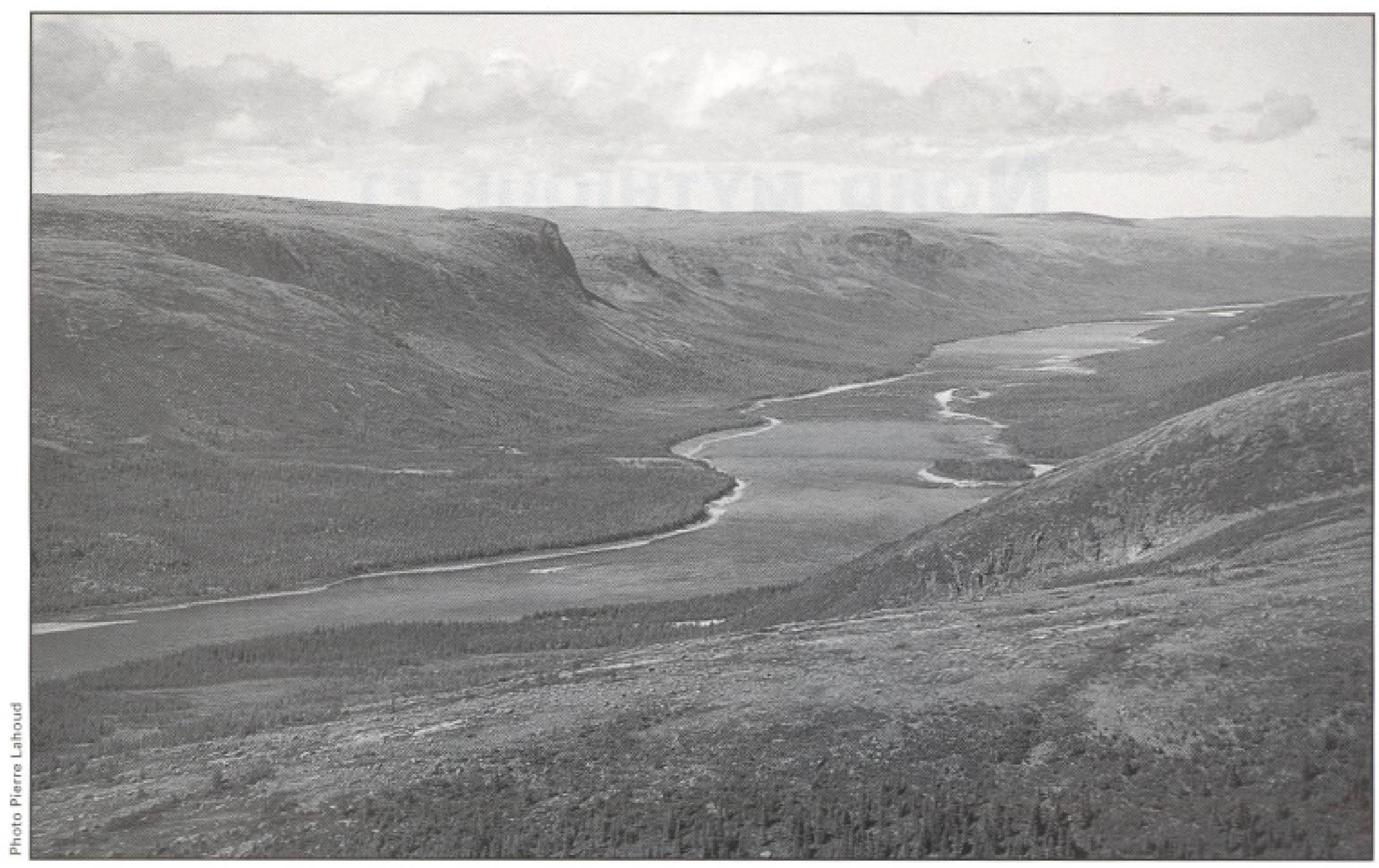

tions européennes, conseient de former une entité culturelle distincte mais vivant en situation bi ou plurilingue., se référant à un territoire d'appartenance mal délimité mais largement aux mains des sociétés dominantes, pratiquant un genre de vie traditionnel, moderne ou une combinaison des deux, désirant la promotion d'institutions locales et régionales en propre à l"intérieur d"un pays (Collectif, 1998),

\section{BIBLIOGRAPHIE}

Arcand, Bernard (1999), Abolissons Thiver!, Montréal, Boréal, $114 \mathrm{p}$.

Arseneau, Max (1999), Notes pour l'allocution du ministre délégué au Tourisne, Québec, Sommet mondial de la nordicité, $24 \mathrm{p}$.

Association canadienne des géographes (1965a), Abstract of Papers [du congres], Vancouver, p. 8.

Association canadienné des géographes (1965b), (s.t.), Revuè de Géographie Alpine, 53 , 4. p. 667 .

Association des villes d'hiver (1999), Reunion a Prince George, Yellowknife.
Cazelais, Normand, et al. (1998), Lespace touristique, Québec, $\mathrm{PUQ}$.

Collectif (1998), L'industrie touristique autochtone $\%$, Téoros, Montréal, 17, 2,62 p.

Collectif (1991), * Mon pays, c'est l'hiver *. Cap-aux-Diamanis, Québec, numéro spécial, $24,78 \mathrm{p}$.

Deffontaines, Pierre (1957), Lhomme el l'hiver au Canada, Paris, Gallimard, 294 p.

de la Soudière, Martin (1987), L'hiver. A la recherche d'une morte saison, Lyon, La Manufacture, 272 p.

Désy, Jean (1967), * La publicité touristique au Moyen Nord [du Québec] w, Bullerin de l'AGAF, 11, p. 73-99.

Hamelin, Louis-Edmond (1974), * Les régions touristiques du Nord canadien w. Bulletin de IAssociation de géographes frangais, 419 , p. 219-228 et traduction par W. Barr dans Canadian Nordicity, Montréal, Harvest House, 1979, p. 272-277.

Hamelin, Louis-Edmond (n.p.), Le Québec par des mots.

Jamet, Heriri (1980), * Le tourisme dans la région de la Baie James et du Nouveau-Québec *, Les Cahiers de I'ACFAS, 1, p. 59-72.
Pressman, Norman (1999), Living in harmony with winter, Prince George, Papyrus, 56 p.

Robitaille, B. et R. Le Jeune (1967), Le potentiel touristique du Nouveau-Québec s, Bulletin de I'AGAF, 11, p. 143-146.

Rudloff, W. (1981), World Climates with Tables..., Stuttgart, $630 \mathrm{p}$.

Toupin, Jerry (1999), Climatologie nivale, Québec, thèse, Universitê Laval, 187 p. + annexes (ms).

Veyret, Paul (1951), wa neige au Canada * Revue de Géographie Alpine, XXXIX. p. 533 . 570.

Wilson, Cynthia (1967-69), Climatology of Cold Regions. Northern Hewisphere, Hanover, CRREL, 141 p. et 158 p. 